\title{
AN APPROXIMATION OF INTEGRABLE FUNCTIONS BY STEP FUNCTIONS WITH AN APPLICATION
}

\author{
M. G. CRANDALL ${ }^{1}$ AND A. PAZY
}

\begin{abstract}
Let $f \in L^{1}(0, \infty), \delta>0$ and $\left(G_{\delta} f\right)(t)=\delta^{-1} \int_{t}^{\infty} e^{(t-s) / \delta} f(s) d s$. Given a partition $P=\left\{0=t_{0}<t_{1}<\cdots<t_{i}<t_{i+1}<\cdots\right\}$ of $[0, \infty)$ where $t_{i} \rightarrow \infty$, we approximate $f$ by the step function $A_{P} f$ defined by

$$
A_{P} f(t)=\left(G_{\delta_{1}} G_{\delta_{i-1}} \cdots G_{\delta_{1}} f\right)(0) \text { for } t_{i-1}<t<t_{i},
$$

where $\delta_{i}=t_{i}-t_{i-1}$. The main results concern several properties of this process, with the most important one being that $A_{P} f \rightarrow f$ in $L^{1}(0, \infty)$ as $\mu(P)=\sup _{i} \delta_{i} \rightarrow 0$. An application to difference approximations of evolution problems is sketched.
\end{abstract}

Introduction. This note is concerned with an interesting method of approximating an integrable function $f:(0, \infty) \rightarrow R$ by step functions. The approximation process involves the integral transformation $G_{\delta}: L^{1}(0, \infty) \rightarrow$ $L^{1}(0, \infty)$ defined for $\delta>0$ by

$$
\left(G_{\delta} f\right)(t)=\frac{1}{\delta} \int_{t}^{\infty} e^{(t-s) / \delta} f(s) d s .
$$

Equivalently, $g=G_{\delta} f$ is the unique function $g \in L^{1}(0, \infty)$ which satisfies $g-\delta g^{\prime}=f$.

Let $P=\left\{0=t_{0}<t_{1}<\cdots<t_{i}<t_{i+1}<\cdots\right\}$ be a partition of $[0, \infty)$ with $\lim _{i \rightarrow \infty} t_{i}=\infty$. The step sizes of the partition are denoted by $\delta_{i}: \delta_{i}=t_{i}-$ $t_{i-1}$. Each partition $P$ determines a piecewise constant approximation $A_{P} f$ of $f$ defined by

$$
A_{P} f(t)=\left(G_{\delta_{i}} G_{\delta_{i-1}} \cdots G_{\delta_{1}} f\right)(0) \text { for } t_{i-1} \leqslant t<t_{i}, i=1,2, \ldots
$$

The mesh of the partition is denoted by $\mu(P) ; \mu(P)=\sup _{1 \leqslant i<\infty} \delta_{i}$. The main results are summarized in the following theorem.

Theorem. Let $P$ be as above, $f \in L^{1}(0, \infty)$ and $A_{P}$ be defined by (2). Then

$$
\begin{gathered}
A_{P} f \in L^{1}(0, \infty), \\
\int_{0}^{\infty}\left|A_{P} f(s)\right| d s \leqslant \int_{0}^{\infty}|f(s)| d s, \\
\int_{0}^{\infty} A_{P} f(s) d s=\int_{0}^{\infty} f(s) d s,
\end{gathered}
$$

Received by the editors August 31, 1978.

AMS (MOS) subject classifications (1970). Primary 41A30.

Key words and phrases. Step functions, approximation theory, accretive operators.

${ }^{1}$ Sponsored by the United States Army under Contract No. DAAG29-75-C-0024 and by the National Science Foundation under Grant No. MCS78 01245. 
and

$$
\lim _{\mu(P) \rightarrow 0} \int_{0}^{\infty}\left|A_{P} f(s)-f(s)\right| d s=0 .
$$

The definition of the transformation $f \rightarrow A_{P} f$ as well as the questions resolved by the theorem arose naturally from considering difference approximations of certain nonlinear evolution problems. While this motivation is not relevant for the statement or the proof (given in \$1) of the theorem, we do explain it briefly in $\$ 2$.

We are indebted to Carl de Boor for his advice on this problem.

1. Proof of the Theorem. Let $h, k \in L^{1}(-\infty, 0)$ and $f \in L^{1}(0, \infty)$. We define $k \circ f \in L^{1}(0, \infty)$ and $h * k \in L^{1}(-\infty, 0)$ according to

$$
(k \circ f)(t)=\int_{t}^{\infty} k(t-s) f(s) d s
$$

and

$$
h * k(r)=\int_{r}^{0} h(r-s) k(s) d s .
$$

The convolution operator "*" is commutative and associative, while

$$
h \circ(k \circ f)=(h * k) \circ f \text {. }
$$

For $\delta>0$ we set

$$
k_{\delta}(r)=\delta^{-1} \exp (r / \delta)
$$

The transformation $G_{\delta}$ in (1) is

$$
G_{\delta} f=k_{\delta} \circ f .
$$

Let $P=\left\{0=t_{0}<t_{1}<\cdots<t_{i}<t_{i+1}<\cdots\right\}$ and $\delta_{i}=t_{i}-t_{i-1}$ be as in the introduction and $A_{P}$ be given by (2). For simplicity of notation we will set

$$
\begin{aligned}
k_{i}=k_{\delta_{i}} \text { and } K_{i}=k_{i} * K_{i-1}=k_{i} * k_{i-1} * \cdots * & k_{1} \\
& \text { for } i=1,2, \ldots
\end{aligned}
$$

Since $k_{i} \geqslant 0, A_{P}$ clearly satisfies

$$
\left|A_{P} f\right| \leqslant A_{P}|f|
$$

Moreover by (2), (1.5), (1.6) and (1.3)

$$
\begin{aligned}
\int_{0}^{t_{i}} A_{P}|f|(s) d s & =\sum_{l=1}^{i} \delta_{l}\left(k_{l} \circ\left(k_{l-1} \circ\left(\cdots \circ\left(k_{2} \circ\left(k_{1} \circ|f|\right)\right) \cdots\right)\right)\right)(0) \\
& =\sum_{l=1}^{i} \delta_{l}\left(K_{l} \circ|f|\right)(0)=\int_{0}^{\infty} \sum_{l=1}^{i} \delta_{l} K_{l}(-s)|f(s)| d s .
\end{aligned}
$$

Since each of the summands $\delta_{l} K_{l}(-s)$ in the last integrand is nonnegative, we can establish (3) and (4) of the theorem by showing that

$$
\sum_{l=1}^{\infty} \delta_{l} K_{l}(r) \leqslant 1 \text { for }-\infty<r<0
$$


while (5) requires

$$
\sum_{l=1}^{\infty} \delta_{l} K_{l}(r)=1 \quad \text { a.e. on }-\infty<r<0 .
$$

The following lemma implies (1.9) since $K_{j} * 1 \geqslant 0$.

LEMMA 1. For each $j=1,2, \ldots$

$$
\sum_{l=1}^{j} \delta_{l} K_{l}+K_{j} * 1 \equiv 1 .
$$

Proof of Lemma 1. We proceed by induction. If $j=1$ the claim is that $\delta_{1} k_{1}+k_{1} * 1 \equiv 1$. Indeed, for any $\delta$,

$$
\delta k_{\delta}+k_{\delta} * 1=e^{r / \delta}+\frac{1}{\delta} \int_{r}^{0} e^{(r-s) / \delta} d s \equiv 1 .
$$

We now assume the claim is true for $j=i$ and verify it for $j=i+1$. By (1.12) we have

$$
\begin{aligned}
K_{i+1} * 1 & =k_{i+1} * K_{i} * 1=k_{i+1} * 1 * K_{i}=\left(1-\delta_{i+1} k_{i+1}\right) * K_{i} \\
& =K_{i} * 1-\delta_{i+1} K_{i+1}=1-\sum_{l=1}^{i+1} \delta_{l} K_{l}
\end{aligned}
$$

where the last equality follows from the induction hypothesis. Rearranging (1.13) yields (1.11) with $j=i+1$ and the proof is complete.

We verify (1.10) indirectly. Let

$$
f_{\sigma}(t)=e^{-\sigma t}
$$

If $\sigma>0$

$$
\left(G_{\delta} f_{\sigma}\right)(t)=\frac{1}{\delta} \int_{t}^{\infty} e^{(t-s) / \delta} e^{-\sigma s} d s=\frac{1}{1+\sigma \delta} e^{-\sigma t}
$$

from which it follows that

$$
\left(A_{P} f_{\sigma}\right)(t)=\prod_{l=1}^{i}\left(1+\sigma \delta_{l}\right)^{-1} \text { for } t_{i-1} \leqslant t<t_{i}
$$

and hence

$$
\int_{0}^{\infty}\left(A_{P} f_{\sigma}\right)(s) d s=\sum_{i=1}^{\infty} \delta_{i} \prod_{l=1}^{i}\left(1+\sigma \delta_{l}\right)^{-1} .
$$

Setting $r_{i}=\left(1+\sigma \delta_{i}\right)^{-1}$ we claim that

$\delta_{1} r_{1}+\delta_{2} r_{2} r_{1}+\cdots+\delta_{i} r_{i} r_{i-1} \cdots r_{2} r_{1}+\sigma^{-1} r_{i} r_{i-1} \cdots r_{2} r_{1}=\sigma^{-1}$

for $i=1,2, \ldots$. The proof parallels the proof of Lemma 1 . Since $\sum_{i=1}^{\infty} \delta_{i}=$ $\infty$, we have $r_{i} r_{i-1} \cdots r_{1} \rightarrow 0$ as $i \rightarrow \infty$ and (1.17), (1.18) together imply

$$
\int_{0}^{\infty}\left(A_{P} f_{\sigma}\right)(s) d s=\frac{1}{\sigma}=\int_{0}^{\infty} f_{\sigma}(s) d s
$$

Setting $f=f_{\sigma}$ in (1.8), letting $i \rightarrow \infty$ and using the above implies (1.10). 
It remains to verify (6). (We remark that the previous results did not require $\mu(P)<\infty$.) In view of $(4)$, which is independent of $P$, it suffices to verify (6) for a dense subset $F$ of $L^{1}(0, \infty)$. It is convenient to choose $F=\operatorname{span}\left\{f_{\sigma}: \sigma>0\right\}$. (In fact, $\operatorname{span}\left\{e^{-n t}: n=1,2, \ldots\right\}$ is dense in $L^{1}(0, \infty)$, as is well known. To see this, use the change of variables $x=e^{-t}$ which exchanges $(0, \infty)$ and $(0,1)$ while $e^{-n t}$ becomes $x^{n}$.) To proceed, we estimate $\left|A_{P} f_{\sigma}-f_{\sigma}\right|$ in terms of $\mu(P)$. For convenience of future referencing the simple lemma which does so is stated without using the notation above.

LEMMA 2. Let $\left\{\delta_{i}\right\}_{i=1}^{\infty}$ be a sequence of positive numbers satisfying $\sum_{i=1}^{\infty} \delta_{i}=$ $\infty$ and $\sigma>0$. Let $t_{0}=0, t_{i}=\delta_{1}+\delta_{2}+\cdots+\delta_{i}$ for $i=1,2, \ldots$ and $\mu=$ $\sup _{1<i<\infty} \delta_{i}$. If

$$
g(t)=\prod_{l=1}^{i}\left(1+\sigma \delta_{l}\right)^{-1} \quad \text { for } t_{i-1}<t<t_{i}
$$

then

$$
\left|g(t)-e^{-\sigma t}\right| \leqslant e^{-\sigma t} \max \left\{e^{\mu \sigma t} e^{\sigma^{2} \mu^{2}}-1,1-e^{-\sigma \mu}\right\} .
$$

Proof of Lemma 2. It is enough to treat $\sigma=1$, for then the general result follows upon replacing $\left\{\delta_{i}\right\}$ by $\left\{\sigma \delta_{i}\right\}$ and $t$ by $\sigma t$. Elementary calculus yields

$$
e^{-\delta} \leqslant(1+\delta)^{-1} \leqslant e^{-\delta+\delta^{2}} \text { for } \delta>0
$$

Multiplying these inequalities yields

$$
e^{-\left(\delta_{1}+\cdots+\delta_{i}\right)} \leqslant \prod_{l=1}^{i}\left(1+\delta_{l}\right)^{-1} \leqslant e^{-\left(\delta_{1}+\cdots+\delta_{l}\right)} e^{\left(\delta_{1}^{2}+\cdots+\delta_{l}^{2}\right)} .
$$

Using (1.20) and the inequalities

$$
\delta_{1}^{2}+\cdots+\delta_{i}^{2} \leqslant \mu\left(\delta_{1}+\cdots+\delta_{i}\right) \text { and } t<\delta_{1}+\cdots+\delta_{i}<t+\mu
$$

for $t_{i-1}<t<t_{i}$ establishes

$$
e^{-t}\left(e^{-\mu}-1\right) \leqslant g(t)-e^{-t} \leqslant e^{-t}\left(e^{\mu(t+\mu)}-1\right)
$$

and hence Lemma 2 in the case $\sigma=1$.

END OF PROOF OF THE THEOREM. By (1.16) and Lemma 2

$$
\int_{0}^{\infty}\left|A_{P} f_{\sigma}-f_{\sigma}\right| d s \leqslant \int_{0}^{\infty} e^{-\sigma s} \max \left\{e^{\mu \sigma s} e^{\sigma^{2} \mu^{2}}-1,1-e^{-\sigma \mu}\right\} d s
$$

where $\mu=\mu(P)$. The right-hand side above tends to zero as $\mu \rightarrow 0$, and the proof is complete.

REMARK. If $m \in L^{1}(-\infty, 0) \cap L^{\infty}(-\infty, 0), m_{\delta}(r)=\delta^{-1} m(r / \delta)$ and (1) is replaced by $G_{\delta} f=m_{\delta} \circ f$, the first part of the above proof adapts to establish that

$$
\int_{0}^{\infty}\left|A_{P} f(s)\right| d s \leqslant C \int_{0}^{\infty}|f(s)| d s
$$

provided $C \geqslant 0$ can be chosen to satisfy

$$
|m(r)|+C \int_{r}^{0}|m(s)| d s \leqslant C \quad \text { a.e. }-\infty<r<0 .
$$


This last estimate is equivalent to $\left|m_{\delta}\right|+\left|m_{\delta}\right| * C \leqslant C$. If $\|m\|_{L^{\prime}(-\infty, 0)}<1$ we may set

$$
C=\|m\|_{L^{\infty}(-\infty, 0)} /\left(1-\|m\|_{L^{1}(-\infty, 0)}\right) .
$$

(Consideration of the case $m(r)=2 e^{r}$ shows some such restriction is necessary.) By contrast, our proof of (5) and (6) used special properties of the exponential kernel.

Motivation. Let $X$ be a Banach space and $A$ be an accretive operator in $X$ (see, e.g., [1], [2] for terminology). If $f \in L^{1}(0, \infty ; X)$ and $x \in X$, we say that

$$
\left\{\begin{array}{l}
u^{\prime}+A u \ni f \\
u(0)=x
\end{array}\right.
$$

has an $\varepsilon$-approximate solution on $[0, T]$ if there are finite sequences $0=t_{0}<$ $t_{1}<\cdots<t_{N}$, and $\left\{f_{1}, f_{2}, \ldots, f_{N}\right\},\left\{x_{0}, x_{1}, \ldots, x_{N}\right\} \subset X$ such that

$$
\frac{x_{i+1}-x_{i}}{t_{i+1}-t_{i}}+A x_{i+1} \ni f_{i+1}, \quad i=0,1, \ldots, N
$$

and

$$
\begin{gathered}
t_{N} \geqslant T, \quad t_{i+1}-t_{i}<\varepsilon, \quad\left\|x_{0}-x\right\|<\varepsilon \quad \text { and } \\
\sum_{i=0}^{N-1} \int_{t_{i}}^{t_{i+1}}\left\|f_{i+1}-f(s)\right\| d s<\varepsilon .
\end{gathered}
$$

In this case, the step function whose value on $\left(t_{i}, t_{i+1}\right]$ is $x_{i+1}$ is called an $\varepsilon$-approximate solution of (2.1). It is shown in [3] that if (2.1) has an $\varepsilon$-approximate solution on $[0, T]$ for each $\varepsilon>0$, then these solutions converge uniformly as $\varepsilon \downarrow 0$ to a unique limit $u \in C([0, T] ; X)$ which is the solution (in a certain sense) of (2.1). The estimates which prove this are considerably more involved in the case that $f \neq 0$ than in the simpler case $f \equiv 0$. The approximation theorem proved in this note allows us to reduce the problem (2.1) with a general $f \in L^{1}(0, \infty ; X)$ to the case $f \equiv 0$ in the following way: Define an operator $\mathbb{Q}$ in $X \times L^{1}(0, \infty ; X)$ by

$$
D(\mathscr{Q})=D(A) \times W^{1,1}(0, \infty ; X)
$$

and

$$
\mathcal{Q}(x, g)=\left\{\left(y-g(0),-g^{\prime}\right): y \in A x\right\} .
$$

We show below that $Q$ is accretive. Given an $\varepsilon$-approximate solution of (2.1) on $[0, T]$ as above, define $\left\{g_{0}, g_{1}, \ldots, g_{N}\right\} \subset W^{1,1}(0, \infty ; X)$ by $g_{0}=f$, $\delta_{i}=t_{i}-t_{i-1}, g_{i+1}=G_{\delta_{i+1}} g_{i}$. Then the function whose value on $\left(t_{i}, t_{i+1}\right]$ is $\left(x_{i+1}, g_{i+1}\right)$ is an

$$
\varepsilon+\sum_{i=0}^{N-1} \int_{t_{i}}^{t_{i+1}}\left\|g_{i+1}(0)-f(s)\right\| d s
$$


approximate solution of

$$
\left\{\begin{array}{l}
\mathscr{L}^{\prime}+\mathscr{Q} \ni 0 \\
\mathscr{U}(0)=(x, f)
\end{array}\right.
$$

By the (clearly valid) version of Theorem 1 in which $L^{1}(0, \infty)$ is replaced by $L^{1}(0, \infty ; X)$ the term

$$
\sum_{i=0}^{N-1} \int_{t_{i}}^{t_{i+1}}\left\|g_{i+1}(0)-f(s)\right\| d s
$$

tends to zero as $\mu=\max _{i} \delta_{i} \rightarrow 0$.

We now briefly sketch the proof that $\mathcal{Q}$ is accretive. If $Z$ is any Banach space, set

$$
[p, q]_{z}=\lim _{\lambda \downarrow 0} \frac{\|p+\lambda q\|_{z}-\|p\|_{z}}{\lambda}=\inf _{\lambda>0} \frac{\|p+\lambda q\|_{z}-\|p\|_{z}}{\lambda} .
$$

If $Y=X \times L^{1}(0, \infty ; X)$ is equipped with the norm

$$
\|(x, g)\|_{Y}=\|x\|_{X}+\int_{0}^{\infty}\|g(s)\|_{X} d s \quad \text { for }(x, g) \in X
$$

then one computes that

$$
[(x, g),(y, h)]_{Y}=[x, y]_{X}+\int_{0}^{\infty}[g(s), h(s)]_{X} d s .
$$

It follows that $\mathcal{Q}$ is accretive in $Y$ if for every $(x, y),(\hat{x}, \hat{y}) \in A$ and $g$, $\hat{g} \in W^{1,1}(0, \infty ; X)$ we have

$$
\begin{aligned}
& {\left[(x-\hat{x}, g-\hat{g}),\left((y-g(0))-(\hat{y}-\hat{g}(0)),-g^{\prime}+\hat{g}^{\prime}\right)\right]_{Y}} \\
& =[x-\hat{x},(y-\hat{y})-(g(0)-\hat{g}(0))]_{X} \\
& \quad+\int_{0}^{\infty}\left[g(s)-\hat{g}(s),-g^{\prime}(s)+\hat{g}^{\prime}(s)\right]_{X} d s>0 .
\end{aligned}
$$

The first term in (2.5) is estimated by

$$
\begin{aligned}
{[x-\hat{x},} & (y-\hat{y})-(g(0)-\hat{g}(0))]_{X} \\
& \geqslant[x-\hat{x},(y-\hat{y})]_{X}-\|g(0)-\hat{g}(0)\|_{X} \\
& \geqslant-\|g(0)-\hat{g}(0)\|_{X},
\end{aligned}
$$

where the first inequality is due to the fact that $[p, q]_{Z}$ is Lipschitz in $q$ with constant 1 and the second inequality is because $(x, y),(\hat{x}, \hat{y}) \in A$ and $A$ is accretive. The second term in (2.5) is given by

$$
\begin{aligned}
\int_{0}^{\infty}[g(s)-\hat{g}(s) & \left.,-g^{\prime}(s)+\hat{g}^{\prime}(s)\right]_{X} d s \\
& =\int_{0}^{\infty}-\frac{d}{d s}\|g(s)-\hat{g}(s)\|_{X} d s=\|g(0)-\hat{g}(0)\|_{X}
\end{aligned}
$$


since

$$
\frac{d}{d s}\|k(s)\|_{X}=\left[k(s), k^{\prime}(s)\right]_{X}
$$

wherever $\|k(s)\|_{X}$ and $k(s)$ are both differentiable.Together, (2.6) and (2.7) imply (2.5) and hence that $Q$ is accretive.

The system (2.4) was introduced in [4] for another purpose. The results we have just obtained reduce many questions concerning (2.1) to the same questions for $f=0$.

\section{REFERENCES}

1. V. Barbu, Nonlinear semigroups and differential equations in Banach spaces, Noordhoff, Leiden, 1976.

2. M. G. Crandall, An introduction to evolution governed by accretive operators, Dynamical Systems: An International Symposium, Vol. 1, Academic Press, New York, 1976, pp. 131-165.

3. M. G. Crandall and L. C. Evans, On the relation of the operator $\partial / \partial s+\partial / \partial \tau$ to evolution governed by accretive operators, Israel J. Math. 21 (1975), 261-278.

4. C. M. Dafermos and M. Slemrod, Asymptotic behaviour of nonlinear contraction semigroups, J. Functional Analysis 13 (1973), 97-106.

Mathematics Department and Mathematics Research Center, University of WISCONSIN-MADISON, MADISON, WISCONSIN 53706

Department of Mathematics, Hebrew University of Jerusalem, Jerusalem, Israel 